\title{
Filosofía en un mundo global
}

\section{Ignacio Ayestarán, Xabier Insausti y Rafael Águila (Editores)}

Editorial Anthropos, Barcelona, 2008, 204 páginas.

\section{Álvaro García*}

Recibida: 09.04.2009

Aceptada: 27.05.2009

$* * *$

Esta obra colectiva presenta un compendio de textos que abordan la problemática de la globalización desde diversos ángulos reflexivos, como la economía, la política, la tecnología o el arte. Se trata de un libro poliédrico en cuanto a su forma, pero profundo en su reflexión filosófica. Sus editores lo han estructurado en cinco secciones pensadas para el ámbito de habla hispana, tanto en América como en Europa:

1) Historia de fenómenos culturales de la globalización

2) Mass media, tecnologías audiovisuales y cibercultura en la comunicación digital

3) Racionalidad, conocimiento y condiciones epistémicas en la globalización

4) Sostenibilidad, ecología y gobernanza en la sociedad del conocimiento global

5) Derechos, utopías y políticas para un mundo global

Los cinco bloques mencionados se van a detallar en los siguientes párrafos para dilucidar el valor de una obra de este estilo, que se sumerge en el reto de pensar la globalización tanto en su vertiente crítica como en su planteamiento funcional.

El primer bloque está orientado hacia una visión reconstructiva de diferentes procesos de globalización que han acaecido a lo largo de la historia. Así el artículo del profesor Javier Aguirre nos transporta a etapas de

\footnotetext{
*Universidad del País Vasco, San Sebastián, España. E-mail: alagar999@hotmail.com
} 
la historia antigua en las que también se vivieron procesos globalizadores. A este respecto nos recuerda el cambio que supuso en la vida griega el paso de la pequeña polis al imperio de Alejandro Magno. Por no hablar del vuelco ideológico que supuso para Europa entera el hecho de que una sola cultura, la romana, se extendiera por todo el continente conectándolo y sometiéndolo a través del sistema reticular de las calzadas romanas.

El profesor Rafael Águila nos hace avanzar un poco más en el tiempo, llevándonos hasta la Edad Media. A finales de esta época, los viejos discursos sobre la Physis, que eliminaban todo signo de individualidad y originalidades del hombre, van cayendo en el olvido. Resurge una nueva forma de pensar, y de pensarse el ser humano, mucho más subjetiva. El ser humano toma conciencia de sí, de ser un sujeto diferenciado de otros sujetos, en un proceso gradual que acabará desembocando en el Renacimiento.

Siguiendo la línea del tiempo llegamos a la confluencia de la Ilustración europea en Hispanoamérica. Xabier Palacios estudia específicamente la expansión del programa liberador en América Latina y sus diferencias: mientras el programa ilustrado europeo se centra en eliminar las diferencias habidas entre los hombres para poder decir más adelante que todos ellos son iguales y que todos tienen la misma dignidad, la ilustración latinoamericana basa sus esfuerzos en remarcar lo que diferencia a unos hombres de otros, a una naciones de otras, en un imparable proceso de independencia.

Cierra este primer bloque el profesor Luis Garagalza acercándonos a los siglos de la modernidad en Europa. Pone de manifiesto la crisis del ideal moderno del progreso, a través del problema del mal como daño adyacente asociado a la idea de bien o a la idea teleológica de la salvación histórica.

El segundo bloque está dedicado a los mass media y a la repercusión que su perfeccionamiento ha tenido sobre la globalización. Sin duda, es impensable que el mundo esté tan eficaz e inmediatamente conectado sin una elaborada red de medios de comunicación. Así, Xavier Puig se detiene a observar la importancia de la imagen en nuestro día a día como elemento de simulación, desprovista completamente de situación espacial o temporal, provocada por la inmediatez y ubicuidad que ofrecen las nuevas tecnologías de la información.

Por otros derroteros va la exposición que realiza Óscar González a propósito de la díada espacio/tiempo en torno al proceso de globalización/ mundialización. Se detiene a explicar que los cambios en los conceptos "espacio" y "tiempo" se han producido otras veces en la historia propiciados por procesos del mismo carácter, sólo que el actual proceso de globalización está marcado por lo que él denomina una "expansión del ser" a través de los mass media.

Gotzon Arrizabalaga hace un repaso a los acontecimientos que, a su 
juicio, han marcado el devenir tanto de la producción musical como de su consumo. Apunta a la deficiente educación musical de una sociedad basada en el consumo inmediato de cualquier producto, incluido el sonoro, y aboga por una reinterpretación del "buen criterio musical”. Señala como causantes de este deterioro del arte musical la capacidad de almacenamiento y modificación de datos que tienen los actuales ordenadores, cuyo buen uso serviría para mejorar y rentabilizar la producción musical.

El fin del bloque corre a cargo de Andoni Alonso, que hace un estudio de los parámetros “globalización”, “tecnociencia” y “cibercultura”, como vectores de un nuevo paradigma sociológico. Aporta gran interés su tesis sobre la interdependencia de esta tríada, resumiéndose de la siguiente manera: la cibercultura en la que vivimos es el producto de una globalización que acaece gracias al desarrollo y las ventajas que aporta la tecnociencia, que a su vez es mejorada por una cibercultura más desarrollada.

La tercera parte del libro está dedicada a la dilucidación de diferentes conceptos que moran en el proceso de globalización, como pueden ser los de racionalidad, universalidad o verdad. El profesor Julián Pacho abre la cuestión argumentando que la ciencia y el conocimiento científico están en la base de la globalización y que son los motores de la misma. Sin embargo la paradoja consiste en que son muy pocos los que conocen de qué manera ayuda la ciencia a tal proceso y muchos los que se benefician de ello. Por tanto, la globalización se ha producido gracias al desconocimiento que la gran mayoría tenemos acerca de cómo funcionan los objetos tecnológicos que la han propiciado, en un juego entre el conocimiento y la ignorancia.

Por su parte, María Albisu se centra en la presentación de las diferentes teorías de la verdad que circulan hoy en día, proponiendo que quizá cooperando unas con otras en el horizonte de un mundo globalizado puedan dar mejores respuestas a la pregunta sobre el valor universal de la verdad dentro de los parámetros de una sociedad mundial o cosmopolita.

El artículo que Xabier Insausti nos presenta reclama reflexionar sobre la condición de lo singular para que se dé lo universal. En un proceso de globalización no sería prudente negar la singularidad, a la par que tampoco es razonable identificarla con lo particular. Se remite a la tesis de que lo singular es a lo particular como lo ontológico es a lo óntico. De otra forma, la globalización acabaría siendo una homogeneización totalitaria.

María Jesús Maidagán ve en el lenguaje poético una forma de escapar de la mediocridad y el empobrecimiento del lenguaje. Ante el vaciamiento de significados de los conceptos, la metáfora aportaría una fuente inagotable de significados que, además, dada su característica universalidad, sus valores podrían traspasar fronteras.

El texto que José Ignacio Galparsoro pone ante nosotros ahonda en la capacidad de la universidad para salvaguardar el honor de la razón, idea 
puramente derridiana. Lo que conseguiría la universidad es no desaparecer en un mundo en el que lo que no tiene valor "preformativo" tiene los días contados. La universidad se presenta por ello como el refugio por excelencia de la razón y la objetividad.

El penúltimo bloque del libro lo vertebran las ideas de sostenibilidad y ecología dentro del marco de la sociedad de la información. El profesor Nicanor Ursua trata el problema de la implantación de un marco éticosocial en el que se desarrollen los acontecimientos de un mundo globalizado, huyendo de visiones unilaterales, y trabajando en la idea de inclusión. En este sentido escruta los factores multicausales convergentes y discute las posibilidades de reforma global, en la línea de Stiglitz.

José Ramón Arana estudia el hecho de que una globalización sostenible y ecológica es necesaria para procurar un presente y un futuro dignos de ser vividos por el ser humano. Para esto se hace completamente necesaria la comprensión de qué es la naturaleza y cómo le puede afectar el proceso de globalización, analizando términos tan necesarios como "habitabilidad”, "lenguaje” e "interacción”.

El profesor Ignacio Ayestarán pone fin a este capítulo haciendo un repaso histórico de la idea de economía a lo largo de los siglos. Deja patente la diferencia entre una economía sostenible-ecológica y la crematística especuladora que rige los mercados bursátiles actuales. Por estas razones enumera diversas claves (espacio, tiempo, globontología, acción, conocimiento y participación) dentro de la crisis de la sociedad del riesgo y de la información mundial.

El bloque que cierra el libro se preocupa por la vertiente política del problema de la globalización, a través de las utopías y los derechos. Por ejemplo, Esteban Antxustegi nos presenta la acuciante necesidad de proponer un sistema normativo-legal que proteja a todos los ciudadanos de la “aldea global”, considerados miembros de una misma especie, con unos mismos valores y una igual dignidad. El objetivo es alcanzar unos derechos universales y cosmopolitas respetando las fronteras nacionales actuales, pero garantizando una mayor justicia y una mayor seguridad a nivel global.

Por su parte, la profesora Belén Altuna nos emplaza en el terreno de las utopías. Ante los enunciados posmodernos sobre “el fin de la historia”, nos llama la atención sobre lo preocupante de pensar que ha llegado el fin de las utopías. Subraya el hecho de que algunas utopías maximalistas han acabado en tragedia, pero argumenta que es necesario llevar a cabo algunas utopías minimalistas que nos podrían ayudar a reorientar nuestro mundo.

El profesor dominicano César Cuello aboga por una globalización alternativa, en la que los valores y las singularidades regionales no se vean afectadas por el neoliberalismo que rige en el proceso globalizador actual. Se centra sobre todo en el caso de América Latina, y reitera que es necesaria una mayor perspectiva intelectual del problema de la globalización, 
dejando atrás la desilusionante sumisión de la que hacen gala los que se someten al imperativo de las grandes firmas y multinacionales.

El último de los textos presentados en el libro corre a cargo del profesor Francisco Javier Caballero. A colación del optimismo mostrado por el norteamericano J. Rifkin acerca de la grandeza de los valores europeos, este profesor hace un repaso crítico a los propósitos económicos en Europa, como aquellos que han propiciado la creación de un mercado común europeo con ciertos efectos negativos o indeseados. De todas formas, sugiere que el planning de futuro de la Europa actual es un camino a seguir para la consecución de un mundo más justo.

En resumen, el libro está atravesado de principio a fin por el problema de la globalización, desde puntos de vista dispares pero complementarios, sujetos al debate público. Constituye así una guía reflexiva y filosófica para los problemas contemporáneos. La lectura de la presente obra ofrece, sin duda, referencias actuales y, sobre todo, una nueva forma de pensar la ciudadanía del siglo XXI. 